\title{
Amelioration of Hepatic Encephalopathy Using Dunaliella salina Microalgae in Rats: Modulation of Hyperammonemia/TLR4
}

\author{
Farouk K. El-Baz $\mathbb{D},{ }^{1}$ Rania Elgohary $\mathbb{D}^{2},{ }^{2}$ and Abeer Salama $\mathbb{D}^{3}$ \\ ${ }^{1}$ Plant Biochemistry Department, National Research Centre (NRC), 33 El Buhouth St., Dokki, Cairo 12622, Egypt \\ ${ }^{2}$ Narcotics, Ergogenics and Poisons Department, National Research Centre (NRC), 33 El Buhouth St., Dokki, Cairo 12622, Egypt \\ ${ }^{3}$ Pharmacology Department, National Research Centre (NRC), 33 El Buhouth St., Dokki, Cairo 12622, Egypt
}

Correspondence should be addressed to Abeer Salama; berrotec@yahoo.com

Received 28 August 2020; Revised 27 February 2021; Accepted 19 March 2021; Published 29 March 2021

Academic Editor: Jane Hanrahan

Copyright (c) 2021 Farouk K. El-Baz et al. This is an open access article distributed under the Creative Commons Attribution License, which permits unrestricted use, distribution, and reproduction in any medium, provided the original work is properly cited.

\begin{abstract}
Hepatic encephalopathy (HE) is a neuropsychiatric disease that is developed as a complication of both acute and chronic liver failure affecting psychomotor dysfunction, memory, and concentration. This study is aimed at evaluating the therapeutic effects of Dunaliella salina (D. salina) microalgae in thioacetamide- (TAA-) induced HE in rats. HE was induced by TAA ( $200 \mathrm{mg} / \mathrm{kg}$; i.p.) for three successive days. Forty male Wister albino rats were divided into 4 groups; the first group was served as a normal, and the second group was injected with TAA and served as TAA control. The third and fourth groups were administered $D$. salina (100 and $200 \mathrm{mg} / \mathrm{kg}$; p.o.), respectively, after TAA injection for 7 days. The behavioral and biochemical markers as well as histological aspects of $\mathrm{HE}$ were estimated. This study revealed that TAA caused behavioral changes, oxidative stress, neuroinflammation, nuclear pyknosis, and neurons degeneration. D. salina improved liver function and decreased oxidative stress and inflammatory mediator as TLR4 protein expression. Also, D. salina elevated HSP-25 and IGF-1 as well as improved brain histopathological alterations. In conclusion, D. salina exerted a therapeutic potential against HE via its antioxidant, antiinflammatory and cytoprotective effects.
\end{abstract}

\section{Introduction}

Hepatic encephalopathy (HE) reflects a spectrum of neuropsychiatric abnormalities such as sensory abnormalities, psychomotor dysfunction, impaired memory, poor concentration, and increased reaction time. These manifestations are due to acute or chronic liver failure [1]. HE affects 45$70 \%$ of patients with liver cirrhosis and $10-20 \%$ of patients with Transjugular Porto Systemic Shunts, in developed countries [2]. HE causes hospitalization and high rates of mortality as a result of end-stage liver disease, reaching to coma [3].

In HE, impaired liver function elevates the levels of ammonia in the blood that crosses the blood-brain barrier and metabolized in the CNS [4]. In brain astrocytes, the ammonia detoxification is due to its incorporation into glutamine ultimately resulting in increased water entry and osmotic forces, astrocytes swelling, and cytotoxic edema
[5]. Hyperammonemia induced inflammation that is not only a partner aggravating the hepatic diseases, but also exacerbates the neuropsychological disturbances in $\mathrm{HE}$ [6]. There is a cross talk between inflammatory mediators and ammonia in HE patients [7] such as toll-like receptors (TLRs), in acute or chronic hepatic diseases, that trigger inflammation [8] and act as critical determinants of $\mathrm{HE}$ severity via releasing proinflammatory mediators [9]. Moreover, TLR4 activation mediates oxidative stress in neurons and hepatocytes [10].

During oxidative stress, in HE patients, heat shock proteins (HSPs) (HSP-25 in rats and HSP27 in humans) have a role as an antioxidant, reducing reactive oxygen species (ROS) and elevating the intracellular glutathione [11]. Also, insulin-like growth factor-1 (IGF-1) is an endocrine growth factor produced in the liver for hepatocytes' growth, differentiation, and proliferation. IGF acts as a hepatoprotective 
against oxidative stress [12]. It improved liver function, suppressed oxidative liver damage, and increased antioxidative enzymes such as catalase, superoxide dismutase, and glutathione peroxidase, in fibrosis and liver cirrhosis rat model [13].

Thioacetamide (TAA), a hepatotoxin, used to induce hepatic failure and $\mathrm{HE}$ in experimental studies due to its efficacy in causing liver and brain disorders similar to human progressive hepatic disorders with brain involvement [14]. TAA undergoes metabolism by the cytochrome P450producing metabolite which in turn responsible for hyperammonemia, oxidative stress, and hepatic necrosis [15].

Previous studies have proven the antioxidant and antiinflammatory activities of different microalgal extracts [16]. Dunaliella salina (D. salina) microalgae are unicellular marine phytoplankton that belongs to the phylum Chlorophyta and family Dunaliellace [17] which contains large amounts of carotenoids at the stationary growth phase and has antioxidant and anticancer activity [18]. It has been shown that $D$. salina carotenoids can preserve hepatic enzyme activity as peroxidase, catalase, and superoxide dismutase which are involved in scavenging ROS [19]. Carotenoids have medicinal properties that are used in several diseases, such as cancer and diabetes, food supplements, cosmetics, and pharmaceuticals. Microalgae therapeutic supplements have importance in the market [20]. Some carotenoids such as astaxanthin, $\beta$-carotene, canthaxanthin, lutein, zeaxanthin, and lycopene are used commercially. $\beta$-Carotene is the pigment carotene, which is the major precursor of vitamin A that has many antioxidant and immune properties [21]. $\beta$-Carotene is trapping radical and considered as an unusual antioxidant [22]. These natural antioxidants when ingested with the diet prevent oxidative stress and induce synergisms [23]. This study was undertaken to investigate the therapeutic effect of the D. salina in TAA-induced HE. In order to give a better insight, we also examined the role of hyperammonemia, oxidative stress, or inflammation pathways by which $D$. salina exerts its therapeutic actions in brain via TLR4, HSP-25, and IGF-1 regulation.

\section{Material and Methods}

2.1. Cultivation of D. salina in the Photobioreactor. Algal species (D. salina) isolated from a salt pond in Al-Fayoum is grown by using Bold nutrient media containing sodium chloride with a concentration of $100 \mathrm{~g} / \mathrm{L}$ for algal isolation and purification [24]. After growing D. salina for 10 days under lab conditions, it was then transferred to a vertical photobioreactor with a capacity of $4000 \mathrm{~L}$. Reservoir (1000 L) tank associated pipework proprietary inline pigging systems. Also, $10 \mathrm{~L}$ basket centrifuge for harvesting was connected to the system. Alga Connect Data Acquisition System was used for online measurements. Tap water was used for the cultivation of algae in the photobioreactor (PBR). Water was sterilized using hypochlorite, and after that, sodium thiosulphate was added to remove the excess hypochlorite. The chlorine test was performed to ensure no residual chlorine is present. The nutrient solution of Bold was used for growing D. salina. One milliliter per liter of micronutrient solution was added to the culture medium. To ensure the purity of the culture, sam- ples are taken regularly and examined microscopically. Carbon dioxide was injected into the culture as a carbon source. The culture is left to grow until the biomass reached the maximum $(2-2.5 \mathrm{~g} / \mathrm{L})$. Algal biomass is harvested using the basket centrifuge at $2000 \mathrm{rpm}$, washed twice with tab water, and dried in the sun dryer where the temperature reached approximately $45^{\circ} \mathrm{C}$ and then grounded into a homogeneous fine powder.

2.2. Chemicals and Kits. Thioacetamide was purchased from Sigma-Aldrich Co., USA. Aspartate aminotransferase (AST), alanine aminotransferase (ALT), ammonia, reduced glutathione (GSH), and malondialdehyde (MDA) were purchased from Biodiagnostic, Cairo, Egypt. Toll-like receptor 4 (TLR4), heat shock protein (HSP-25), and insulin growth factor-1 (IGF-1) were purchased from NOVA, Beijing, China.

2.3. Animals. Forty male Wistar albino rats weighing 120 $150 \mathrm{~g}$ were obtained from the animal house of the National Research Centre (Dokki, Cairo, Egypt) and were kept in standard polypropylene cages under standard environmental conditions with equal light-dark cycles. Rats were adapted for 1 week and were fed rat normal pellet diet and water ad libitum, before the beginning of the experiment. This experiment was carried out in accordance with the Ethics Committee of the National Research Centre, Egypt, and followed the National Institutes of Health Guide Recommendations Care and Use of Laboratory Animals (Publication No. 85-23, revised 1985).

2.4. Experimental Design. Rats were divided into 4 groups; the first group was injected with saline $(2.5 \mathrm{ml} / \mathrm{kg}$; i.p.) for three successive days and received distilled water $(5 \mathrm{ml} / \mathrm{kg}$; p.o.) for 7 days to be considered as a normal, while the second group injected with TAA ( $200 \mathrm{mg} / \mathrm{kg}$; i.p.) for three successive days to induce $\mathrm{HE}$ and served as TAA control [25]. The third and fourth groups were administered $D$. salina (100 and $200 \mathrm{mg} / \mathrm{kg}$; p.o.), respectively [26, 27], after TAA injection for 7 days.

2.5. Behavioral Test (Rotarod Test). Motor coordination of rats was assessed using an accelerating rotarod (Model No. 7750; Ugo Basile), according to the procedure described by [28]. Rats were given three training sessions on three successive days. All rats were trained on the rotarod apparatus at a fixed speed of 4 rotations per minute (rpm) to reach a stable performance, before starting treatment with $D$. salina. On the fourth day, the rats were placed on the testing rod and the speed of the rotarod started at $4 \mathrm{rpm}$ and then increased gradually to reach $40 \mathrm{rpm}$ over $300 \mathrm{~s}$. The basal falling latency time for each rat was recorded using a cutoff limit of $300 \mathrm{~s}$. After the last administration of $D$. salina, each rat was then replaced on the accelerating rotarod apparatus for $300 \mathrm{~s}$ test sessions and the final falling latency time was recorded [29].

2.6. Serum Biochemical Analysis for Liver Enzyme and Ammonia. At the end of the experiment, rats were anesthetized with pentobarbital sodium and blood samples were withdrawn from the retroorbital venous plexus. Collected 
blood samples were allowed to stand for $10 \mathrm{~min}$ at room temperature then centrifuged at $4^{\circ} \mathrm{C}$ using a cooling centrifuge (Laborezentrifugen, 2k15, Sigma, Germany) at $3000 \mathrm{rpm}$ for $10 \mathrm{~min}$ [30]. Sera were separated for assessment of AST, ALT, and ammonia [31].

2.7. Preparation of Tissue Homogenate. The brain was then excised, washed with saline, and placed in ice-cold phosphate buffer (pH 7.4) using a tissue homogenizer (MPW-120, Bit-Lab Medical instruments, Poland) to prepare $20 \%$ homogenate. Homogenized tissues were centrifuged at $4000 \mathrm{rpm} / \mathrm{min}$ for $10 \mathrm{~min}$ at $4^{\circ} \mathrm{C}$ using a cooling centrifuge (Laboratory Centrifuge, 2K15, Sigma Co., Germany). The supernatant was collected and stored at $-80^{\circ} \mathrm{C}$ [32] and then used for the estimation of brain contents of TLR4, HSP-25, and IGF-1.

2.8. Assessment of Oxidative Stress. Lipid peroxides were estimated as thiobarbituric acid-reactive substances (TBARS) [33]. The absorbance of the resulting pink color was measured at $532 \mathrm{~nm}$. The serum level of reduced glutathione (GSH) was determined. Both protein and nonprotein thiol $(-\mathrm{SH})$ groups (mainly GSH) react with Ellman's reagent [5,5-dithiobis (2-nitrobenzoic acid)] to form a stable yellow color of 5-mercapto-2-nitrobenzoic acid, which can be measured colorimetrically at $412 \mathrm{~nm}$ [34].

2.9. Assessment of Brain Contents of TLR4, HSP-25, and IGF-1. Brain contents of TLR4, HSP-25, and IGF-1 were determined using ELISA (Enzyme-Linked Immunosorbent Assay) kit. We followed the manufacturer's instructions of the NOVA kit, Beijing, China, for calculating the results. Standards and samples were pipetted into wells with immobilized antibodies specific for rat TLR4, HSP-25, and IGF-1 and then were incubated. After incubation and washing, biotinylated antirat TLR4, HSP-25, and IGF-1 antibodies were added. Any unbound substances were washed away; horseradish peroxidase-conjugated streptavidin was pipetted into the wells, which were washed once again. TMB (tetramethylbenzidine) substrate solution was added to the wells; color developed proportionally to the amount of TLR4, HSP-25, and IGF-1 bound. Color development was discontinued (Stop Solution), and its intensity was measured at $450 \mathrm{~nm}$.

2.10. Statistical Analysis. All the values are presented as means \pm standard error of the means (SE). Data were evaluated by one-way analysis of variance followed by Tukey's multiple comparisons test. The Graph pad Prism software, version 5 (Inc., San Diego, USA) was used to carry out these statistical tests. The difference was considered significant when $p<0.05$.

2.11. Histopathological Examination of Brain Tissue. Brain tissues were harvested from the different groups and fixed in $10 \%$ neutral buffered formalin. The brain tissues were processed to obtain $4 \mu \mathrm{m}$ paraffin-embedded sections then stained with hematoxylin and eosin stain (H\&E). To determine an appropriate scoring system for brain tissue changes, the scores were derived semiquantitatively using light microscopy.

\section{Results}

3.1. Effect of D. salina on the Behavioral Test (Rotarod Test). The induction of HE by TAA ( $200 \mathrm{mg} / \mathrm{kg}$; i.p.) for three alternative days revealed a significant reduction in the final falling downtime by $65 \%$ when compared to the normal. Oral administration of D. salina (100 and $200 \mathrm{mg} / \mathrm{kg}$ ) for 7 consecutive days significantly elevated final falling downtime by $61 \%$ and $125 \%$, respectively, as compared to the TAA control (Figure 1).

3.2. Effect of D. salina on Liver Enzymes and Serum Ammonia. TAA injection resulted in a significant rise in serum ALT, AST, and ammonia levels by $83 \%, 72 \%$, and $134 \%$, respectively, when compared to the normal. Posttreatment with D. salina (100 and $200 \mathrm{mg} / \mathrm{kg}$ ) for 7 consecutive days decreased serum levels of ALT by $28 \%$ and $44 \%$ and serum AST by $10 \%$ and $21 \%$, as well as ammonia by $19 \%$ and $39 \%$, respectively, in comparison to TAA control. Also, treatment with D. salina $(200 \mathrm{mg} / \mathrm{kg})$ returned serum ALT level to the normal value (Table 1 ).

3.3. Effect of D. salina on Oxidative Stress. As depicted in Table 2, HE induced by TAA significantly increased MDA serum level by $99 \%$ and decreased GSH serum level by $58 \%$ when compared to normal. Posttreatment with D. salina (100 and $200 \mathrm{mg} / \mathrm{kg}$ ) significantly ameliorated serum MDA level by $20 \%$ and $35 \%$, respectively, and elevated serum GSH level by $94 \%$ and $112 \%$, respectively, when compared to TAA control. Moreover, treatment with $D$. salina $(200 \mathrm{mg} / \mathrm{kg})$ restored the MDA level to normal value.

3.4. Effect of D. salina on TLR4, HSP-25, and IGF-1. Brain TLR4 content was significantly elevated in the TAA control by 1 fold when compared to normal. Oral treatment with D. salina $(200 \mathrm{mg} / \mathrm{kg})$ significantly reduced TLR 4 content by $45 \%$ and returned it to normal value, while $D$. salina $(100 \mathrm{mg} / \mathrm{kg})$ did not reduce it when compared to the TAA control (Figure 2).

TAA injection significantly decreased brain HSP-25 content by $61 \%$ when compared to normal. D. salina (100 and $200 \mathrm{mg} / \mathrm{kg}$ ) posttreatment increased HSP-25 brain content by $38 \%$ and $121 \%$, respectively, when compared to the TAA control. Moreover, treatment with D. salina $(200 \mathrm{mg} / \mathrm{kg})$ restored HSP-25 to the normal value (Figure 3).

Induction of $\mathrm{HE}$ produced a decrease in brain content of IGF- 1 by $80 \%$ when compared to normal, while the administration of $D$. salina at a dose of $200 \mathrm{mg} / \mathrm{kg}$ increased it by 1.79 folds. However, the administration of D. salina at a dose of $100 \mathrm{mg} / \mathrm{kg}$ did not change it, when compared to the TAA control (Figure 4).

\subsection{Histopathological Findings}

3.5.1. Effects of D. salina Powder on the Cerebral Cortex. Normal group showed no histopathological alteration and the normal histological structure of the neurons was recorded in cerebral cortex. TAA control showed nuclear pyknosis and degeneration in most of the neurons (red arrow). Both 


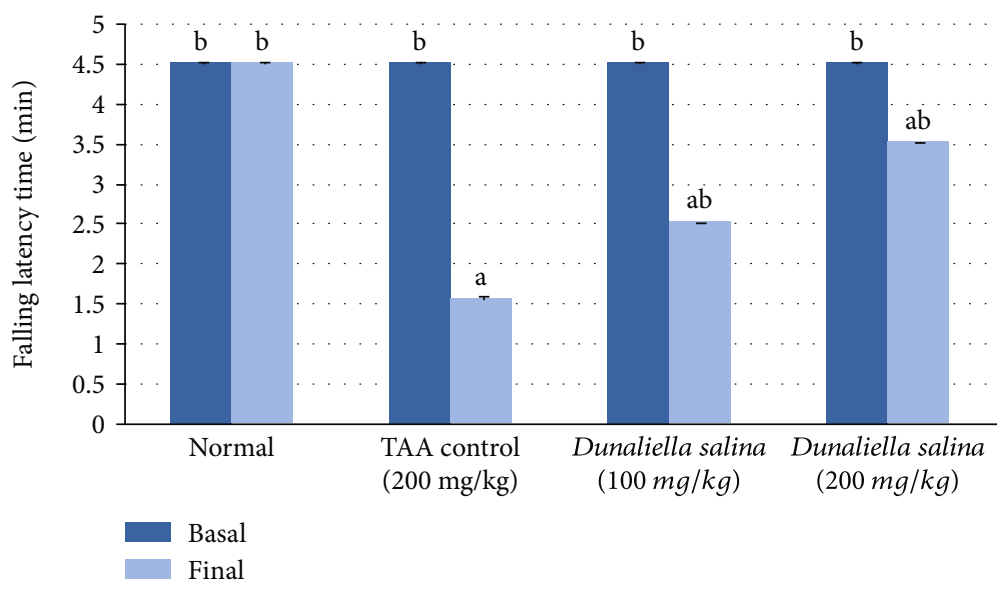

FIGURE 1: Effects of D. salina powder on the behavioral test (rotarod test). Data are presented as the mean \pm SE of $n=10$ for each group. Statistical analysis was carried out by one-way analysis of variance followed by Tukey's multiple comparisons test. ${ }^{a}$ Statistically significant from normal group. ${ }^{\mathrm{b}}$ Statistically significant from the TAA control at $p<0.05$.

TABLE 1: Effects of D. salina powder on serum hepatic functions biomarkers and ammonia.

\begin{tabular}{ccccc}
\hline & Normal & TAA control $(200 \mathrm{mg} / \mathrm{kg})$ & Dunaliella salina $(100 \mathrm{mg} / \mathrm{kg})$ & Dunaliella salina $(200 \mathrm{mg} / \mathrm{kg})$ \\
\hline ALT (U/L) & $40.35 \pm 0.36^{\mathrm{b}}$ & $73.98 \pm 0.88^{\mathrm{a}}$ & $53.10 \pm 0.63^{\mathrm{ab}}$ & $41.42 \pm 0.23^{\mathrm{b}}$ \\
$\%$ of TAA & & $100 \%$ & $72 \%$ & $56 \%$ \\
AST (U/L) & $63.22 \pm 0.42^{\mathrm{b}}$ & $108.68 \pm 0.68^{\mathrm{a}}$ & $98.20 \pm 0.31^{\mathrm{ab}}$ & $85.85 \pm 0.54^{\mathrm{ab}}$ \\
$\%$ of TAA & & $100 \%$ & $90 \%$ & $79 \%$ \\
Ammonia $(\mu \mathrm{mol} / \mathrm{L})$ & $135.80 \pm 0.26^{\mathrm{b}}$ & $317.80 \pm 4.04^{\mathrm{a}}$ & $257.80 \pm 3.39^{\mathrm{ab}}$ & $195.20 \pm 0.33^{\mathrm{ab}}$ \\
$\%$ of TAA & & $100 \%$ & $81 \%$ & $61 \%$ \\
\hline
\end{tabular}

Data are presented as the mean \pm SE of $n=10$ for each group and presented as $\%$ of the TAA control. Statistical analysis was carried out by one-way analysis of variance followed by Tukey's multiple comparisons test. ${ }^{\mathrm{a}}$ Statistically significant from normal group. ${ }^{\mathrm{b}}$ Statistically significant from the TAA control at $p<0.05$.

TABLE 2: Effects of D. salina powder on serum oxidative stress biomarkers.

\begin{tabular}{ccccc}
\hline & Normal & TAA control $(200 \mathrm{mg} / \mathrm{kg})$ & Dunaliella salina $(100 \mathrm{mg} / \mathrm{kg})$ & Dunaliella salina $(200 \mathrm{mg} / \mathrm{kg})$ \\
\hline GSH $(\mathrm{mg} / \mathrm{dl})$ & $15.56 \pm 0.03^{\mathrm{b}}$ & $6.53 \pm 0.06^{\mathrm{a}}$ & $12.67 \pm 0.08^{\mathrm{ab}}$ & $13.87 \pm 0.03^{\mathrm{ab}}$ \\
$\%$ of TAA & & $100 \%$ & $194 \%$ & $212 \%$ \\
MDA $(\mathrm{nmol} / \mathrm{ml})$ & $9.74 \pm 0.07^{\mathrm{b}}$ & $19.36 \pm 0.29^{\mathrm{a}}$ & $15.43 \pm 0.07^{\mathrm{ab}}$ & $12.56 \pm 0.10^{\mathrm{b}}$ \\
$\%$ of TAA & & $100 \%$ & $80 \%$ & $65 \%$ \\
\hline
\end{tabular}

Data are presented as the mean \pm SE of $n=10$ for each group and presented as \% of the TAA control. Statistical analysis was carried out by one-way analysis of variance followed by Tukey's multiple comparisons test. ${ }^{a}$ Statistically significant from normal group. ${ }^{b}$ Statistically significant from the TAA control at $p<0.05$.

doses of $D$. salina showed no histopathological alteration (Figure 5).

3.5.2. Effects of D. salina Powder on the Hippocampus. Normal group showed no histopathological alteration, and the normal histological structure of the neurons was recorded in the subiculum and fascia dentata and hilus. TAA control showed nuclear pyknosis and degeneration in most of the neurons in the subiculum and the subiculum, fascia dentata, and hilus (red arrow). A low dose of D. salina showed no histopathological alteration in the subiculum. A low dose of $D$. salina showed nuclear pyknosis and degeneration in some neurons in the subiculum, fascia dentata, and hilus (red arrow). High dose of $D$. salina showed no histopathological alteration as recorded in the subiculum and fascia dentata and hilus (Figure 6).
3.5.3. Effects of D. salina Powder on the Striatum. Normal showed no histopathological alteration and the normal histological structure of the neurons was recorded in striatum. TAA control showed diffuse gliosis (yellow arrow) and focal hemorrhage (black arrow) in between the nuclear pyknosis and degenerated neurons (red arrow). A low dose of D. salina showed diffuse gliosis (yellow arrow) was noticed in between the nuclear pyknotic nuclear and degenerated neurons and another intact one (red arrow). A high dose of D. salina showed no histopathological alteration (Figure 7). The severity of alteration in the brain was blindly scored microscopically, and scores are presented in Table 3.

\section{Discussion}

Hepatic encephalopathy (HE) is a syndrome arising from acute or chronic liver diseases and is considered as a 


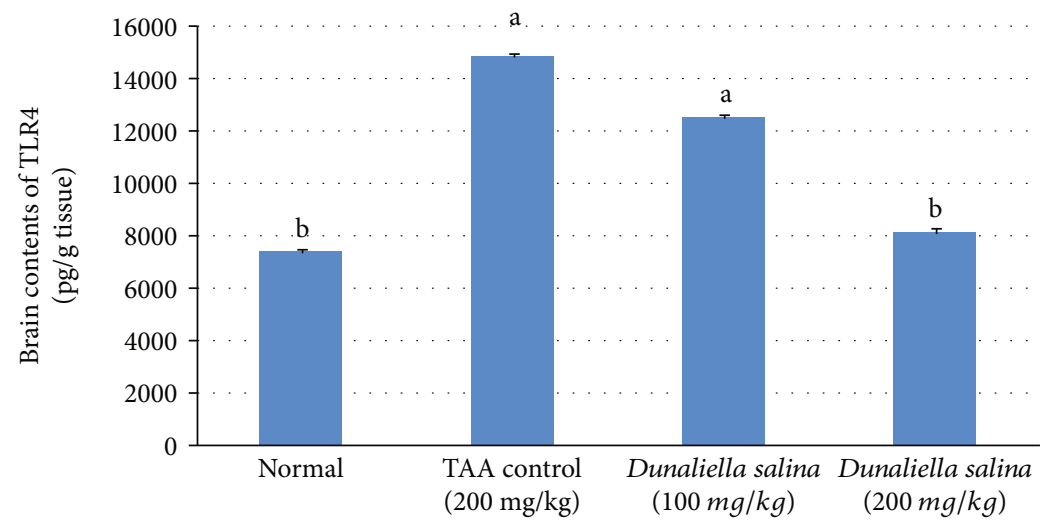

FIGURE 2: Effects of D. salina powder on hepatic contents of TLR4. Data are presented as the mean \pm SE of $n=10$ for each group. Statistical

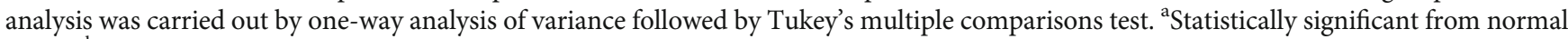
group. ${ }^{\mathrm{b}}$ Statistically significant from the TAA control at $p<0.05$.

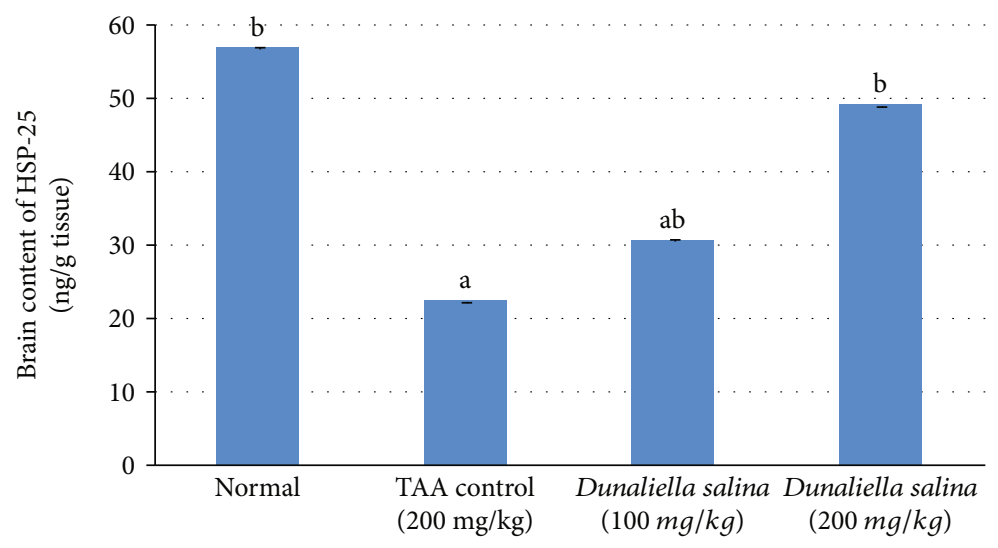

FIGURE 3: Effects of D. salina powder on hepatic contents of HSP-2. Data are presented as the mean \pm SE of $n=10$ for each group. Statistical

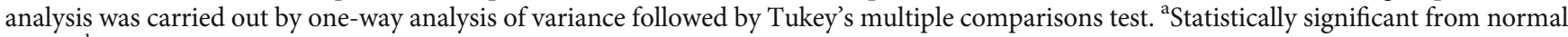
group. ${ }^{\mathrm{b}}$ Statistically significant from the TAA control at $p<0.05$.

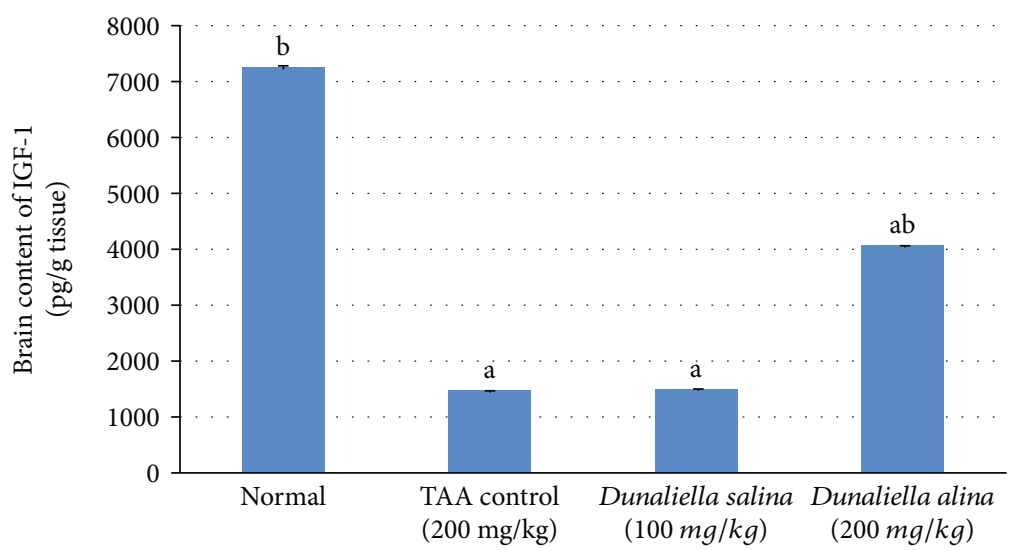

Figure 4: Effects of D. salina powder on hepatic contents of IGF-1. Data are presented as the mean \pm SE of $n=10$ for each group. Statistical

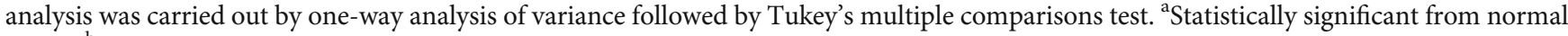
group. 'Statistically significant from the TAA control at $p<0.05$.

neuropsychiatric complication [35]. In the present work, the results showed that thioacetamide (TAA) induced a state of liver dysfunction linked with brain affection. Animals that injected with TAA showed a significant elevation in AST and ALT activities with a concomitant rise in ammonia level.
TAA is a hepatotoxin inducing hepatic failure and elevating serum levels of AST and ALT [36] which expressed in hepatocyte cytoplasm. They are entering the bloodstream after hepatocyte injury as the cell membrane permeability increases. Their elevation reflects the degree of hepatocyte 


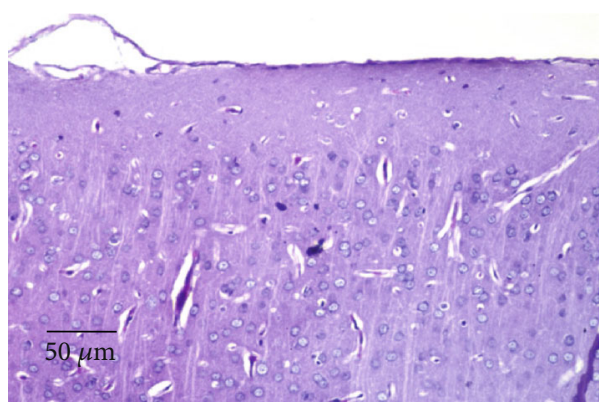

(a)

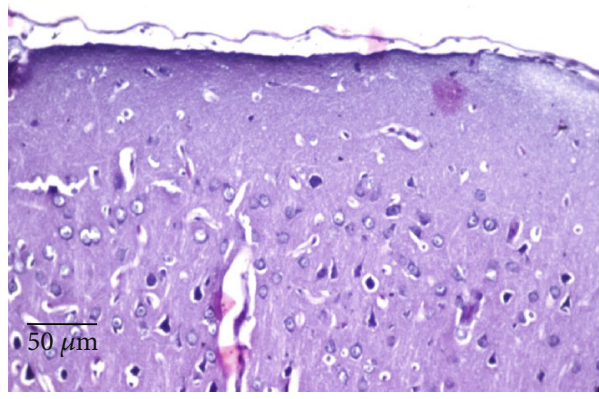

(c)

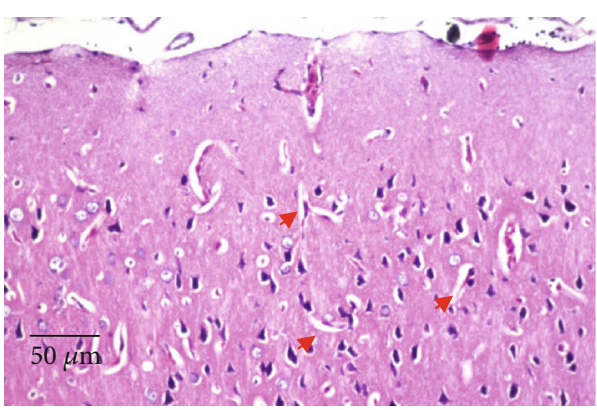

(b)

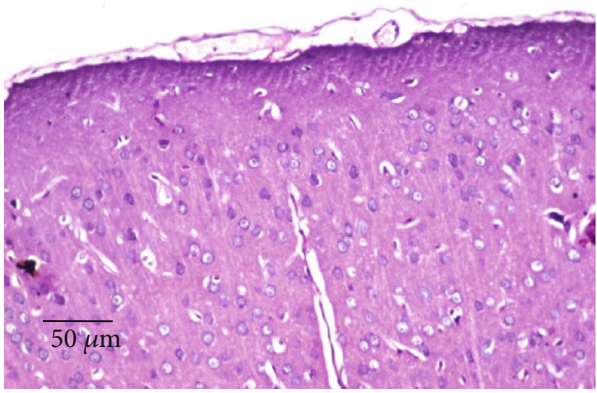

(d)

Figure 5: Effects of D. salina powder on cerebral cortex. (a) Normal showed no histopathological alteration and the normal histological structure of the neurons. (b) TAA control showed nuclear pyknosis and degeneration in most of the neurons (red arrow). (c) A low dose of $D$. salina showed no histopathological alteration. (e) A high dose of D. salina showed no histopathological alteration (H\&E stain, $\times 200$ scale bar $50 \mu \mathrm{m})$.

damage [37]. There was a significant increase; also, in the blood, ammonia level was observed in the TAA control, compared to the normal, in a previous study [38]. This hyperammonemia is due to liver insufficient detoxification and the reduced urinary loss of ammonia leading to direct ammonia provoked neurotoxicity [39]. On the other hand, rats have given both doses of $D$. salina for 7 consecutive days significantly decreased serum activities of ALT and AST, as well as ammonia level, compared to TAA control. These results indicate the beneficial role of $D$. salina in restoring liver integrity. In line with these results, our previous work showed that the administration of $D$. salina for 1 month decreased serum activities of ALT and AST and has an antifibrotic effect against TAA-induced liver fibrosis [40]. Moreover, treatment with $D$. salina preserved hepatocyte integrity [40].

Hyperammonemia-induced neuroinflammation activated cerebellar astrocytes and microglia and produced cerebellum motor coordination dysfunction [41]. In HE patients, there was subclinical motor slowing and impaired visuoconstructive ability, visual perception, and mild cognitive dysfunction [42]. In our study, TAA injection exhibited a significant decline in the final falling downtime in the rotarod test due to motor slowing and dysfunction compared to normal. This result is in line with a previous study [43]. However, oral administration of both doses of $D$. salina significantly increased the final falling downtime as compared to the TAA control as a result of decreasing ammonia brain level and suggesting that D. salina restored motor and cognitive functions, especially high dose.
A close correlation between ammonia level elevation and toll-like receptor (TLR4) upregulation is found in astrocytes and endothelial cells (ECs), under neuroinflammatory conditions, and worsen HE $[44,45]$. TLR4 protein is one of the common factors responsible for inflammatory mediators release from brain ECs and microglia after ammonia exposure resulting in astrocyte swelling. TLR4 stimulates nuclear factor-kappa $\mathrm{B}(\mathrm{NF}-\kappa \mathrm{B})$ that releases proinflammatory cytokines tumour necrosis factor-alpha (TNF- $\alpha$ ), interleukin 1 beta (IL-1 $\beta$ ), and interleukin 6 (IL-6) which is involved in immune responses $[46,47]$. Together with ammonia, TNF$\alpha$ upregulation in astrocytes affects neuronal survival, learning, and memory [48]. In the present work, TAA induced liver dysfunction and hyperammonemia that associated with enhanced expression of TLR4 brain content as compared to normal rats (Figure 8). In HE, TAA caused brain TLR4 elevation in mice [49], while the oral administration of the high dose of $D$. salina, only, was significantly reduced brain TLR4 content as compared to its normal value, suggesting antiinflammatory effects of its components; $\beta$-carotene and zeaxanthin.

Increased ammonia, also, produced astrocyte swelling, reactive nitrogen, and oxygen radical formation, lipid peroxidation elevation [50], and reduction of the activity of antioxidant enzymes in the brain $[51,52]$ and amplifies the neuronal derangements [53]. In the present work, TAAinduced hyperammonemia reduced glutathione (GSH) and elevated malondialdehyde (MDA) serum levels as compared to control rats. On the other hand, oral treatment especially with a high dose of $D$. salina elevated serum GSH level and 


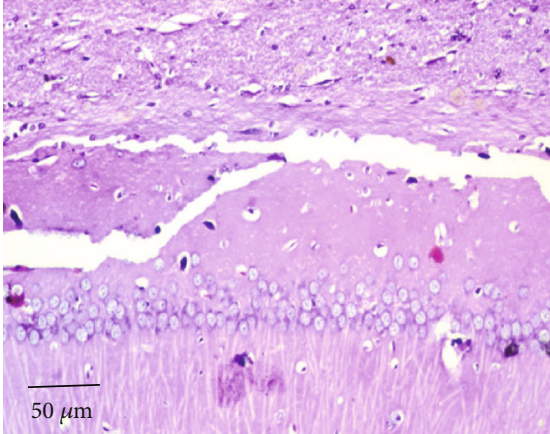

(a)

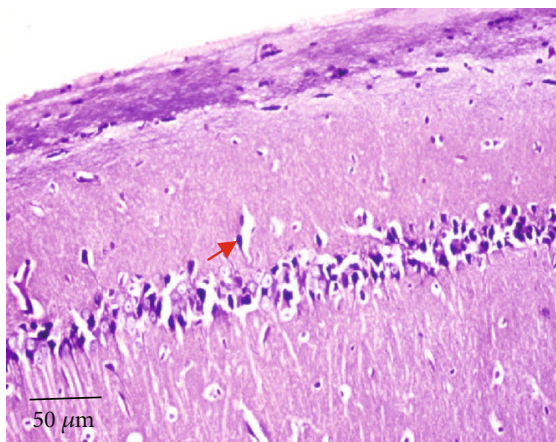

(c)

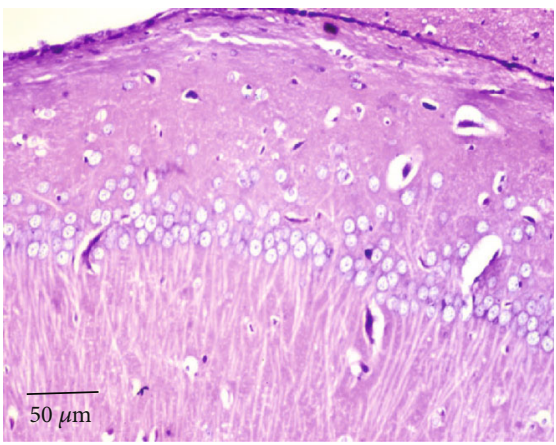

(e)

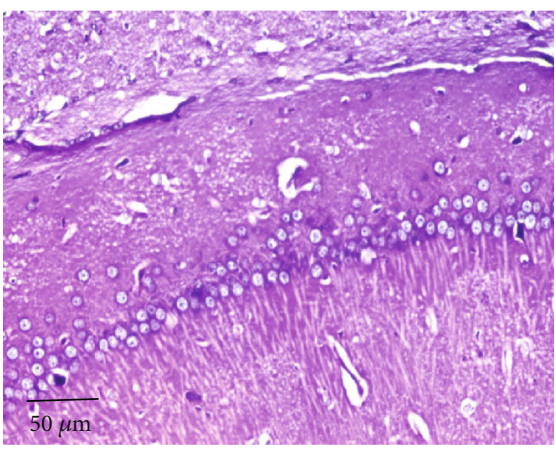

(g)

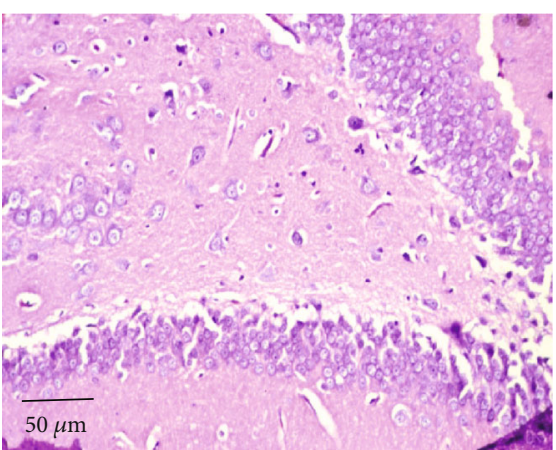

(b)

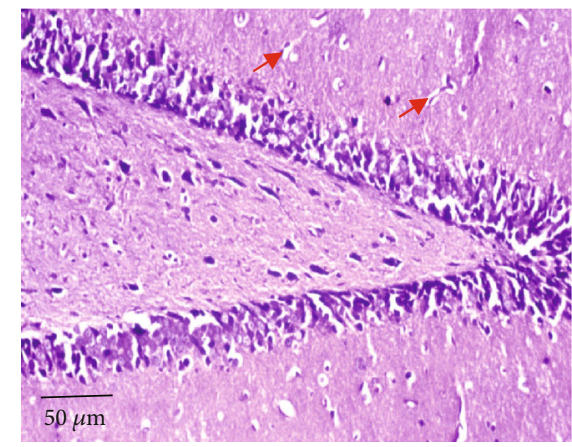

(d)

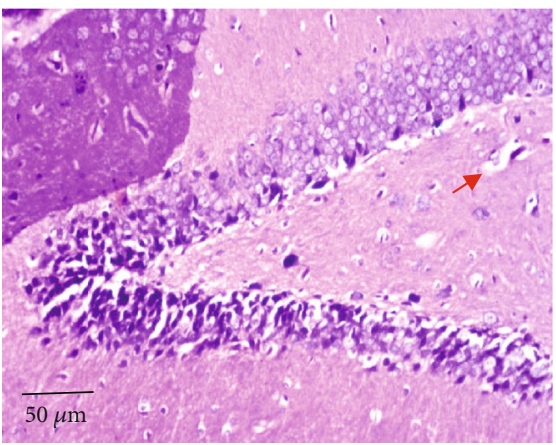

(f)

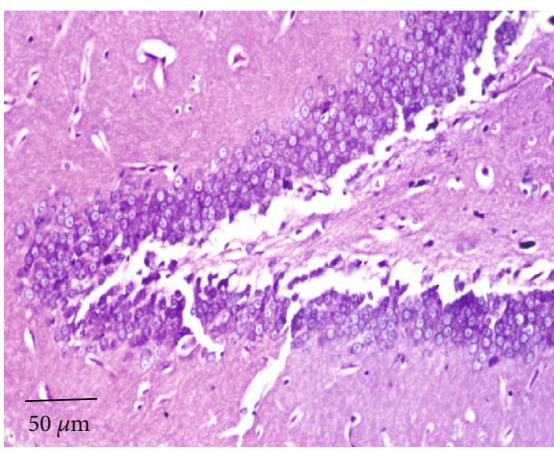

(h)

FIgURE 6: Effects of $D$. salina powder on the hippocampus. (a) Normal showed no histopathological alteration and the normal histological structure of the neurons was recorded in the subiculum. (b) Normal showed no histopathological alteration and the normal histological structure of the neurons was recorded in fascia dentata and hilus. (c) TAA control showed nuclear pyknosis and degeneration in most of the neurons in the subiculum (red arrow). (d) TAA group showed nuclear pyknosis and degeneration in most of the neurons in fascia dentata and hilus (red arrow). (e) A low dose of D. salina showed no histopathological alteration in the subiculum. (f) A low dose of $D$. salina showed nuclear pyknosis and degeneration in some neurons in fascia dentata and hilus (red arrow). (g) High dose of D. salina showed no histopathological alteration as recorded in the subiculum. (h) A high dose of D. salina showed no histopathological alteration in fascia dentata and hilus (H\&E stain, $\times 200$ scale bar $50 \mu \mathrm{m}$ ). 


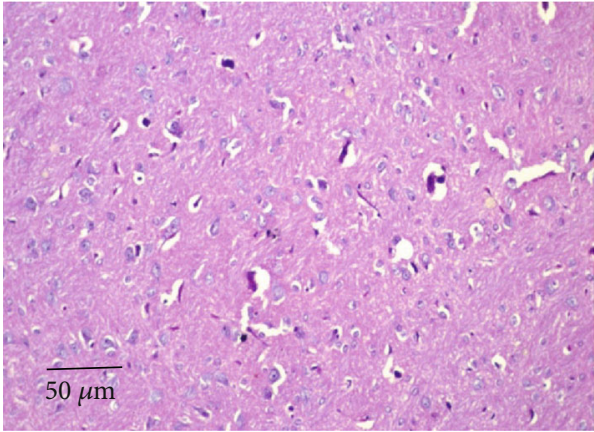

(a)

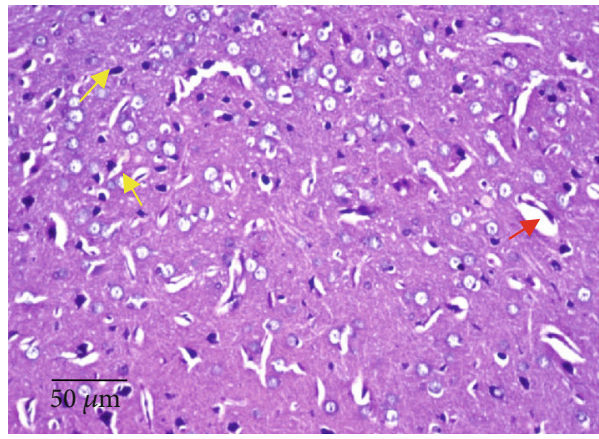

(c)

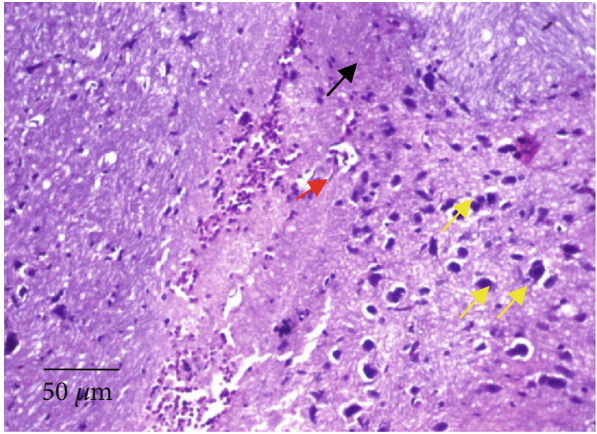

(b)

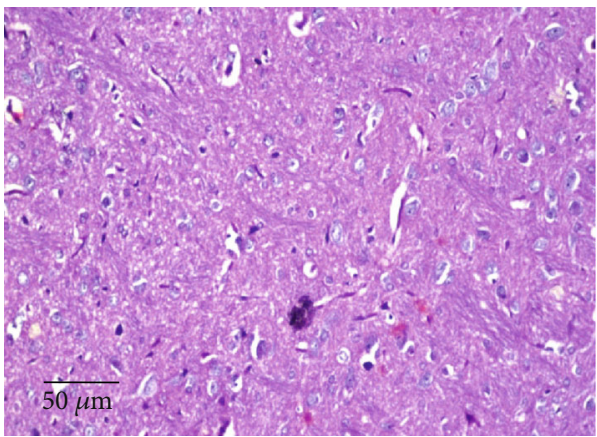

(d)

FIgURE 7: Effects of D. salina powder on striatum: (a) normal showed no histopathological alteration and the normal histological structure of the neurons was recorded. (b) TAA control showed diffuse gliosis (yellow arrow) and focal hemorrhage (black arrow) in between the nuclear pyknosis and degenerated neurons (red arrow). (c) A low dose of D. salina showed diffuse gliosis (yellow arrow) was noticed in between the nuclear pyknotic nuclear and degenerated neurons and another intact one (red arrow). (d) A high dose of D. salina showed no histopathological alteration (H\&E stain, $\times 200$ scale bar $50 \mu \mathrm{m}$ ).

TABLE 3: Effects of D. salina powder on histopathological scoring in brain tissue.

\begin{tabular}{|c|c|c|c|c|c|}
\hline & & Normal & $\begin{array}{l}\text { TAA control } \\
(200 \mathrm{mg} / \mathrm{kg})\end{array}$ & $\begin{array}{l}\text { Dunaliella salina } \\
\quad(100 \mathrm{mg} / \mathrm{kg})\end{array}$ & $\begin{array}{l}\text { Dunaliella salina } \\
(200 \mathrm{mg} / \mathrm{kg})\end{array}$ \\
\hline $\begin{array}{l}\text { Cerebral } \\
\text { cortex }\end{array}$ & $\begin{array}{c}\text { Nuclear pyknosis and neuronal } \\
\text { degeneration }\end{array}$ & - & +++ & - & - \\
\hline \multirow[t]{2}{*}{ Hippocampus } & $\begin{array}{c}\text { Nuclear pyknosis and neuronal } \\
\text { degeneration }\end{array}$ & - & ++ & + & - \\
\hline & Focal hemorrhage & - & ++ & - & - \\
\hline \multirow[t]{2}{*}{ Striatum } & $\begin{array}{c}\text { Nuclear pyknosis and neuronal } \\
\text { degeneration }\end{array}$ & - & +++ & ++ & - \\
\hline & Gliosis & - & ++ & + & - \\
\hline
\end{tabular}

+++: sever histopatholocical alteration. ++: moderate histopatholocical alteration. +: mild histopatholocical alteration. -: nil histopatholocical alteration.

returned MDA level to its normal value. These results may suggest that $D$. salina is a potent anti-inflammatory and antioxidant agent in the nervous system that suppresses inflammatory and oxidative stress pathways through its carotenoid contents such as zeaxanthin and $\beta$-carotene, treating HE. Indeed, recent studies explored that carotenoids inhibit NF- $\kappa$ B activity [54] regulating inflammation-related and oxidative stress genes in neurodegenerative diseases [55]. Also, marine carotenoids have antioxidant properties by activation of the antioxidant network (GSH and catalase) [56] and scavenging reactive oxygen species (ROS) [57]. D. salina, in another study, regulates oxidative stress and protects the liver from fibrosis [16].
Increased cellular stress induces the expression of heat shock proteins (HSPs) affecting neurons' response [58]. HSP72 and HSP 25/27 play a role in cellular protection [59]. HSP-25 downregulates 6-hydroxydopamine-induced cytochrome c release and apoptosis and protects complex I activity during oxidative stress. Thus, elevated HSP-25 expression is a defense mechanism for neuronal cells under stress conditions, via its antioxidant properties [60]. Increased HSP expression is linked to attenuation of the proinflammatory cytokines as well as oxidative stress [61]. Regarding, TAA injection significantly induced brain oxidative stress and inflammation associated with decreased brain HSP-25 content when compared to normal rats. The p38 


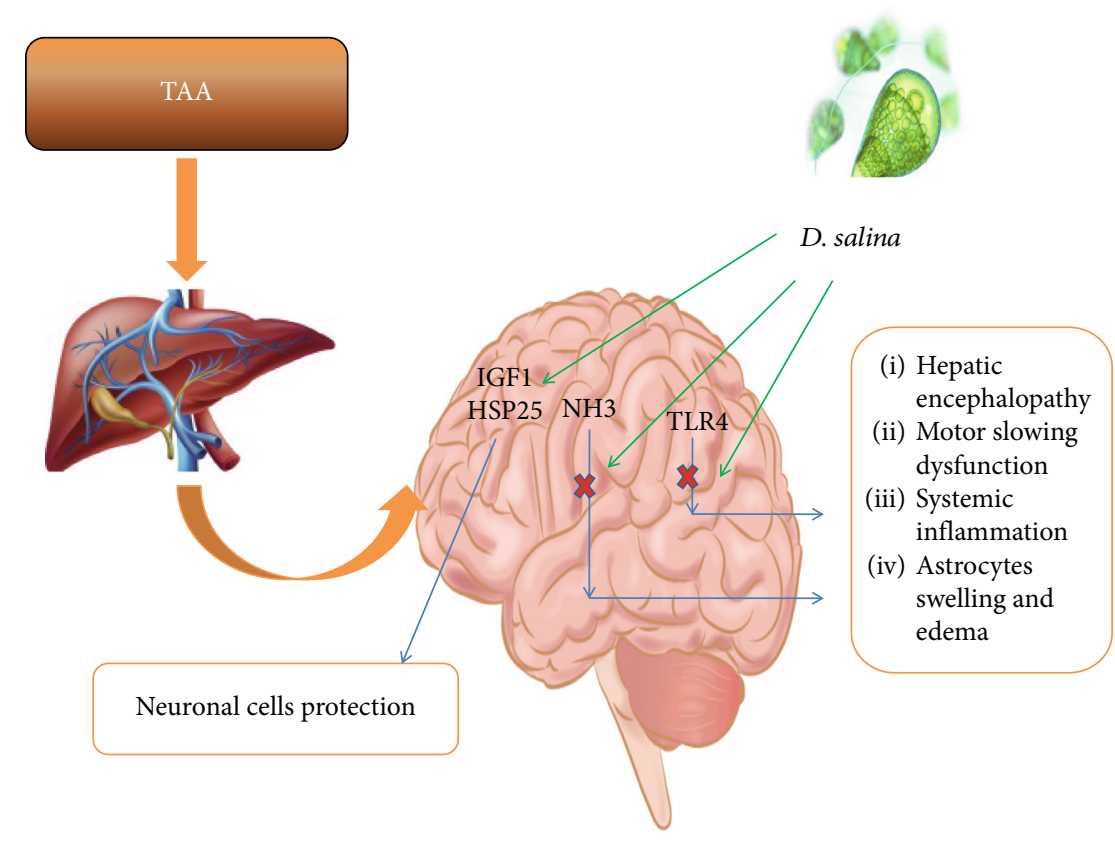

Figure 8: Effects of D. salina powder on hepatic encephalopathy through hyperammonemia/TLR4 pathway.

mitogen-activated protein kinase (MAPK) isoform is upregulated in response to oxidative stress or inflammation which in turn modulates antioxidants, inflammatory mediators, and survival gene expression [62]. p38a controls the expression of HSP-25 preventing oxidative stress. Restoration of HSP-25 expression plays an important role in TAA-induced ROS accumulation and fibrogenesis [63]. In another study, TAA injection showed an inverse correlation between HSP-25 expression and ROS release in mice [64]. Both doses of D. salina posttreatment enhanced HSP-25 brain content when compared to the TAA control exerting defense mechanism for neuronal cells against oxidative damage through the potant antioxidant activity of $\beta$-carotene and zeaxanthin, especially high dose.

Neurodegeneration development, also, is influenced by insulin-like growth factor-1 (IGF-1) disruption. IGF-1 plays a vital role in the regulation of growth and metabolism [65]. IGF-1 inhibits brain oxidative insults [66] and protects neurons against the diverse effect of oxidative stress $[67,68]$. In our study, the induction of $\mathrm{HE}$ produced a decrease in brain content of IGF-1 with nuclear pyknosis and degeneration in most of the neurons, when compared to normal rats. In an agreement with our study, $\mathrm{HE}$ induced by azoxymethane suppressed IGF-1 expression [69] and produced nuclear pyknosis and neuron degeneration [70]. This study, for the first time, showed the effect of $D$. salina on IGF-1 in brain tissue. Administration of a high dose of $D$. salina caused a significant increase in brain content of IGF-1, when compared to the TAA control and inhibited nuclear pyknosis and neuron degeneration induced by TAA, indicating its therapeutic role in $\mathrm{HE}$.

\section{Conclusion}

D. salina has antioxidative and anti-inflammatory activities in the brain. It controls liver function, ammonia, and behav- ioral changes. Also, D. salina regulates lipid peroxidation, an antioxidant enzyme, TLR4, HSP-25, and IGF-1, exhibiting neurotherapeutic activity, so further works are needed to examine its clinical benefits in brain pathologies associated with oxidative stress and inflammation.

\section{Data Availability}

The data used to support the findings of this study are available from the corresponding author upon request.

\section{Conflicts of Interest}

The authors declare that there are no conflicts of interest.

\section{Authors' Contributions}

Farouk K. El-Baz contributed to the conceptualization, writing, and editing. Rania Elgohary participated in the methodology, formal analysis, and writing-original draft. Abeer A.A. Salama contributed to the conceptualization, methodology, formal analysis, investigation, and writing-review and editing.

\section{References}

[1] P. Ferenci, A. Lockwood, K. Mullen, R. Tarter, K. Weissenborn, and A. T. Blei, "Hepatic encephalopathy-definition, nomenclature, diagnosis, and quantification: final report of the working party at the 11th World Congresses of Gastroenterology, Vienna, 1998," Hepatology, vol. 35, no. 3, pp. 716-721, 2002.

[2] K. C. Raphael and S. S. Matuja, "Hepatic encephalopathy; prevalence, precipitating factors and challenges of management in a resource-limited setting," Journal of Gastrointestinal \& Digestive System, vol. 6, no. 3, pp. 441-446, 2016. 
[3] M. L. Volk, R. S. Tocco, J. Bazick, M. O. Rakoski, and A. S. Lok, "Hospital readmissions among patients with decompensated cirrhosis," The American Journal of Gastroenterology, vol. 107, no. 2, pp. 247-252, 2012.

[4] D. Suraweera, V. Sundaram, and S. Saab, "Evaluation and management of hepatic encephalopathy: current status and future directions," Gut and liver, vol. 10, no. 4, pp. 509-519, 2016.

[5] M. Romero-Gómez, S. Montagnese, and R. Jalan, "Hepatic encephalopathy in patients with acute decompensation of cirrhosis and acute-on-chronic liver failure," Journal of Hepatology, vol. 62, no. 2, pp. 437-447, 2015.

[6] D. L. Shawcross, N. A. Davies, R. Williams, and R. Jalan, "Systemic inflammatory response exacerbates the neuropsychological effects of induced hyperammonemia in cirrhosis," Journal of Hepatology, vol. 40, no. 2, pp. 247-254, 2004.

[7] L. Jain, B. C. Sharma, P. Sharma, S. Srivastava, A. Agrawal, and S. K. Sarin, "Serum endotoxin and inflammatory mediators in patients with cirrhosis and hepatic encephalopathy," Digestive and Liver Disease, vol. 44, no. 12, pp. 1027-1031, 2012.

[8] H. Luo, J. Wang, C. Qiao, N. Ma, D. Liu, and W. Zhang, "Pycnogenol attenuates atherosclerosis by regulating lipid metabolism through the TLR4-NF- $\kappa \mathrm{B}$ pathway," Experimental of Molecular Medicine, vol. 47, no. 10, pp. e191-e191, 2015.

[9] W.-W. Wang, Y. Zhang, X.-B. Huang, N. You, L. Zheng, and J. Li, "Fecal microbiota transplantation prevents hepatic encephalopathy in rats with carbon tetrachloride-induced acute hepatic dysfunction," World Journal of Gastroenterology, vol. 23, no. 38, pp. 6983-6994, 2017.

[10] F. Hua, J. Ma, T. Ha et al., "Activation of Toll-like receptor 4 signaling contributes to hippocampal neuronal death following global cerebral ischemia/reperfusion," Journal of Neuroimmunology, vol. 190, no. 1-2, pp. 101-111, 2007.

[11] A. Vidyasagar, N. A. Wilson, and A. Djamali, "Heat shock protein 27 (HSP27): biomarker of disease and therapeutic target," Fibrogenesis \& Tissue Repair, vol. 5, no. 1, p. 7, 2012.

[12] C. Postic, R. Dentin, and J. Girard, "Role of the liver in the control of carbohydrate and lipid homeostasis," Diabetes \& Metabolism, vol. 30, no. 5, pp. 398-408, 2004.

[13] H. M. Brown-Borg, S. G. Rakoczy, M. A. Romanick, and M. A. Kennedy, "Effects of growth hormone and insulin-like growth factor-1 on hepatocyte antioxidative enzymes," Experimental Biology and Medicine, vol. 227, no. 2, pp. 94-104, 2016.

[14] L. C. D. Lima, A. S. Miranda, R. N. Ferreira, M. A. Rachid, and A. C. S. de Silva, "Hepatic encephalopathy: lessons from preclinical studies," World Journal of Hepatology, vol. 11, no. 2, pp. 173-185, 2019.

[15] I. Swapna, K. V. Kumar, P. Reddy, C. Murthy, P. Reddanna, and B. Senthilkumaran, "Phospholipid and cholesterol alterations accompany structural disarray in myelin membrane of rats with hepatic encephalopathy induced by thioacetamide," Neurochemistry International, vol. 49, no. 3, pp. 238-244, 2006.

[16] F. K. El-Baz, A. A. A. Salama, and R. A. Hussein, "Dunaliella salina microalgae oppose thioacetamide-induced hepatic fibrosis in rats," Toxicology Reports, vol. 7, pp. 36-45, 2020.

[17] A. Hosseini Tafreshi and M. Shariati, "Dunaliella biotechnology: methods and applications," Journal of Applied Microbiology, vol. 107, no. 1, pp. 14-35, 2009.

[18] H. Zamani, B. Rastegari, and M. Varamini, "Antioxidant and anticancer activity of Dunaliella salina extract and oral drug delivery potential via nano-based formulations of gum Arabic coated magnetite nanoparticles," Journal of Drug Delivery Science and Technology, vol. 54, p. 101278, 2019.

[19] P. Singh, M. Baranwal, and S. M. Reddy, "Antioxidant and cytotoxic activity of carotenes produced by Dunaliella salina under stress," Pharmaceutical Biology, vol. 54, no. 10, pp. 2269-2275, 2016.

[20] I. Priyadarshani and B. Rath, "Commercial and industrial applications of micro algae-a review," Journal of Algal Biomass Utilization, vol. 3, no. 4, pp. 89-100, 2012.

[21] R. Sathasivam and J.-S. Ki, "A review of the biological activities of microalgal carotenoids and their potential use in healthcare and cosmetic industries," Marine Drugs, vol. 16, no. 1, p. 26, 2018.

[22] K. C. Murthy, A. Vanitha, J. Rajesha, M. M. Swamy, P. Sowmya, and G. A. Ravishankar, "In vivo antioxidant activity of carotenoids from Dunaliella salina- a green microalga," Life Sciences, vol. 76, no. 12, pp. 1381-1390, 2005.

[23] Y.-W. Hsu, C.-F. Tsai, W.-H. Chang, Y.-C. Ho, W.-K. Chen, and F.-J. Lu, "Protective effects of Dunaliella salina-a carotenoid-rich alga, against carbon tetrachloride-induced hepatotoxicity in mice," Food and Chemical Toxicology, vol. 46, no. 10, pp. 3311-3317, 2008.

[24] J. E. Stein, Handbook of Phycological Methods. Culture Methods and Growth Measurements, Cambridge University Press, 1975.

[25] M. A. El-Latif El-Ghazaly, E. R. Rashed, G. M. Shafey, H. F. Zaki, and A. S. Attia, "Amelioration of thioacetamideinduced hepatic encephalopathy in rats by low-dose gamma irradiation," Environmental Science and Pollution Research, vol. 27, no. 1, pp. 334-343, 2020.

[26] F. K. El-Baz, A. Salama, and R. A. A. Salama, "Dunaliella salina attenuates diabetic neuropathy induced by STZ in rats: involvement of thioredoxin," BioMed Research International, vol. 2020, Article ID 1295492, 11 pages, 2020.

[27] F. K. El-Baz, H. F. Aly, and A. A. A. Salama, "Toxicity assessment of the green Dunaliella salina microalgae," Toxicology Reports, vol. 6, pp. 850-861, 2019.

[28] R. Vijitruth, M. Liu, D.-Y. Choi, X. V. Nguyen, R. L. Hunter, and G. Bing, "Cyclooxygenase-2 mediates microglial activation and secondary dopaminergic cell death in the mouse MPTP model of Parkinson's disease," Journal of Neuroinflammation, vol. 3, no. 1, p. 6, 2006.

[29] A. H. Salama, H. Elmotasem, and A. A. A. Salama, "Nanotechnology-based blended chitosan-pectin hybrid for safe and efficient consolidative antiemetic and neuroprotective effect of meclizine hydrochloride in chemotherapy-induced emesis," International Journal of Pharmaceutics, vol. 584, p. 119411, 2020.

[30] S. M. El-Dakhly, A. A. A. Salama, S. O. M. Hassanin, N. N. Yassen, A. A. Hamza, and A. Amin, "Aescin and diosmin each alone or in low dose- combination ameliorate liver damage induced by carbon tetrachloride in rats," BMC Research Notes, vol. 13, no. 1, p. 259, 2020.

[31] D. F. Mansour, A. A. Salama, R. R. Hegazy, E. A. Omara, and S. A. Nada, "Whey protein isolate protects against cyclophosphamide-induced acute liver and kidney damage in rats," Journal of Applied Pharmaceutical Science, vol. 7, no. 6, pp. 111-120, 2017.

[32] H. Elmotasem, H. K. Farag, and A. A. Salama, "In vitro and in vivo evaluation of an oral sustained release hepatoprotective 
caffeine loaded w/o Pickering emulsion formula - Containing wheat germ oil and stabilized by magnesium oxide nanoparticles," International Journal of Pharmaceutics, vol. 547, no. 1-2, pp. 83-96, 2018.

[33] M. B. Ruiz-Larrea, A. M. Leal, M. Liza, M. Lacort, and H. de Groot, "Antioxidant effects of estradiol and 2hydroxyestradiol on iron-induced lipid peroxidation of rat liver microsomes," Steroids, vol. 59, no. 6, pp. 383-388, 1994.

[34] E. Beutler, O. Duron, and B. M. Kelly, "Improved method for the determination of blood glutathione," The Journal of Laboratory and Clinical Medicine, vol. 61, pp. 882-888, 1963.

[35] J. Lizardi-Cervera, P. Almeda, L. Guevara, and M. Uribe, "Hepatic encephalopathy: a review," Annals of Hepatology, vol. 2, no. 3, pp. 122-130, 2003.

[36] N. A. Afifi, A. Ramadan, W. El-Eraky, A. A. A. Salama, A. A. El-Fadaly, and A. Hassan, "Quercetin protects against thioacetamide-induced hepatotoxicity in rats through decreased oxidative stress biomarkers, the inflammatory cytokines; (TNF- $\alpha$ ), (NF- $\kappa$ B) and DNA fragmentation," Der Pharma Chemica, vol. 8, no. 9, pp. 48-55, 2016.

[37] J. Zheng, X. Tian, W. Zhang et al., "Protective effects of fucoxanthin against alcoholic liver injury by activation of Nrf2Mediated antioxidant defense and inhibition of TLR4mediated inflammation," Marine Drugs, vol. 17, no. 10, p. 552, 2019.

[38] E. Fadillioglu, C. Gursul, and M. Iraz, "Effects of caffeic acid phenethyl ester on thioacetamide-induced hepatic encephalopathy in rats," Progress in Neuro-Psychopharmacology and Biological Psychiatry, vol. 34, no. 8, pp. 1440-1445, 2010.

[39] F. Nicolao, C. Efrati, A. Masini, M. Merli, A. F. Attili, and O. Riggio, "Role of determination of partial pressure of ammonia in cirrhotic patients with and without hepatic encephalopathy," Journal of Hepatology, vol. 38, no. 4, pp. 441-446, 2003.

[40] F. K. El-Baz, A. Salama, and R. A. A. Salama, "Therapeutic effect of Dunaliella salina microalgae on thioacetamide(TAA-) induced hepatic liver fibrosis in rats: role of TGF- $\beta$ and MMP9," BioMed Research International, vol. 2019, Article ID 7028314, 9 pages, 2019.

[41] V. Hernandez-Rabaza, A. Cabrera-Pastor, L. Taoro-Gonzalez et al., "Neuroinflammation increases GABAergic tone and impairs cognitive and motor function in hyperammonemia by increasing GAT-3 membrane expression. Reversal by sulforaphane by promoting M2 polarization of microglia," Journal of Neuroinflammation, vol. 13, no. 1, p. 83, 2016.

[42] C. Montoliu, R. Rodrigo, P. Monfort et al., "Cyclic GMP pathways in hepatic encephalopathy. Neurological and therapeutic implications," Metabolic Brain Disease, vol. 25, no. 1, pp. 3948, 2010.

[43] S. Singh and S. Trigun, "Low grade cirrhosis induces cognitive impairment and motor dysfunction in rats: could be a model for minimal hepatic encephalopathy," Neuroscience Letters, vol. 559, pp. 136-140, 2014.

[44] A. R. Jayakumar, X. Y. Tong, K. M. Curtis, R. Ruiz-Cordero, M. T. Abreu, and M. D. Norenberg, "Increased toll-like receptor 4 in cerebral endothelial cells contributes to the astrocyte swelling and brain edema in acute hepatic encephalopathy," Journal of Neurochemistry, vol. 128, no. 6, pp. 890-903, 2014.

[45] R.-S. Lin, F.-Y. Lee, S.-D. Lee et al., "Endotoxemia in patients with chronic liver diseases: relationship to severity of liver diseases, presence of esophaegeal varices, and hyperdynamic circulation," Journal of Hepatology, vol. 22, no. 2, pp. 165-172, 1995.
[46] E. Lorne, H. Dupont, and E. Abraham, “Toll-like receptors 2 and 4: initiators of non-septic inflammation in critical care medicine?," Intensive Care Medicine, vol. 36, no. 11, pp. 1826-1835, 2010.

[47] V. Makó, J. Czúcz, Z. Weiszhár et al., "Proinflammatory activation pattern of human umbilical vein endothelial cells induced by IL- $1 \beta$, TNF- $\alpha$, and LPS," Cytometry Part A, vol. 77, no. 10, pp. 962-970, 2010.

[48] H. R. Frederiksen, H. Haukedal, and K. Freude, "Cell type specific expression of toll-like receptors in human brains and implications in Alzheimer's disease," BioMed Research International, vol. 2019, Article ID 7420189, 18 pages, 2019.

[49] D. H. El-Kashef and M. S. Serrya, "Sitagliptin ameliorates thioacetamide-induced acute liver injury via modulating TLR4/NF-KB signaling pathway in mice," Life Sciences, vol. 228, pp. 266-273, 2019.

[50] P. V. B. Reddy, C. R. Murthy, and P. Reddanna, "Fulminant hepatic failure induced oxidative stress in nonsynaptic mitochondria of cerebral cortex in rats," Neuroscience Letters, vol. 368, no. 1, pp. 15-20, 2004.

[51] K. V. Rama Rao, A. R. Jayakumar, and M. D. Norenberg, "Brain edema in acute liver failure: mechanisms and concepts," Metabolic Brain Disease, vol. 29, no. 4, pp. 927-936, 2014.

[52] M. Y. Alomar and A. M. Al-Attar, "Effect of basil leaves extract on liver fibrosis induced by thioacetamide in male rats," International Journal of Pharmacology, vol. 15, no. 4, pp. 478-485, 2019.

[53] D. Häussinger and B. Görg, "Interaction of oxidative stress, astrocyte swelling, and cerebral ammonia toxicity," Current Opinion in Clinical Nutrition \& Metabolic Care, vol. 13, no. 1, pp. 87-92, 2010.

[54] T. Liu, W.-h. Liu, J.-s. Zhao, F.-z. Meng, and H. Wang, "Lutein protects against $\beta$-amyloid peptide-induced oxidative stress in cerebrovascular endothelial cells through modulation of Nrf-2 and NF- $\kappa$ b," Cell Biology and Toxicology, vol. 33, no. 1, pp. 5767, 2017.

[55] E. M. Conner and M. B. Grisham, "Inflammation, free radicals, and antioxidants," Nutrition, vol. 12, no. 4, pp. 274-277, 1996.

[56] C.-T. Shiu and T.-M. Lee, "Ultraviolet-B-induced oxidative stress and responses of the ascorbate-glutathione cycle in a marine macroalga Ulva fasciata," Journal of Experimental Botany, vol. 56, no. 421, pp. 2851-2865, 2005.

[57] J. Fiedor and K. Burda, "Potential role of carotenoids as antioxidants in human health and disease," Nutrients, vol. 6, no. 2, pp. 466-488, 2014.

[58] V. Calabrese, G. Scapagnini, A. Ravagna et al., "Increased expression of heat shock proteins in rat brain during aging: relationship with mitochondrial function and glutathione redox state," Mechanisms of Ageing and Development, vol. 125, no. 4, pp. 325-335, 2004.

[59] A. M. De, "Heat shock proteins: facts, thoughts, and dreams," Shock, vol. 11, no. 1, pp. 1-12, 1999.

[60] A. M. Gorman, E. Szegezdi, D. J. Quigney, and A. Samali, "Hsp27 inhibits 6-hydroxydopamine-induced cytochrome c release and apoptosis in PC12 cells," Biochemical and Biophysical Research Communications, vol. 327, no. 3, pp. 801-810, 2005.

[61] C.-G. Yoo, S. Lee, C.-T. Lee, Y. W. Kim, S. K. Han, and Y.S. Shim, "Anti-inflammatory effect of heat shock protein induction is related to stabilization of $\mathrm{I} \kappa \mathrm{B} \alpha$ through preventing 
$\mathrm{I} \kappa \mathrm{B}$ kinase activation in respiratory epithelial cells," The Journal of Immunology, vol. 164, no. 10, pp. 5416-5423, 2000.

[62] E. F. Wagner and Á. R. Nebreda, "Signal integration by JNK and p38 MAPK pathways in cancer development," Nature Reviews Cancer, vol. 9, no. 8, pp. 537-549, 2009.

[63] T. Sakurai, M. Kudo, A. Umemura et al., "p38 $\alpha$ inhibits liver fibrogenesis and consequent hepatocarcinogenesis by curtailing accumulation of reactive oxygen species," Cancer Research, vol. 73, no. 1, pp. 215-224, 2013.

[64] C. Garrido, M. Brunet, C. Didelot, Y. Zermati, E. Schmitt, and G. Kroemer, "Heat shock proteins 27 and 70: anti-apoptotic proteins with tumorigenic properties," Cell Cycle, vol. 5, no. 22, pp. 2592-2601, 2006.

[65] S. Fernandez, A. Fernandez, C. Lopez-Lopez, and I. TorresAleman, "Emerging roles of insulin-like growth factor-I in the adult brain," Growth Hormone \& IGF Research, vol. 17, no. 2, pp. 89-95, 2007.

[66] E. Williams, S. Grant, A. Petrescu et al., "P: 5 Let7f expression is upregulated in the frontal cortex during acute liver failure and contributes to hepatic encephalopathy via downregulation of IGF1 expression," American Journal of Gastroenterology, vol. 114, no. 1, pp. S2-S3, 2019.

[67] S. Heck, F. Lezoualc'h, S. Engert, and C. Behl, "Insulin-like Growth Factor-1-mediated Neuroprotection against Oxidative Stress Is Associated with Activation of Nuclear Factor $\kappa \mathrm{B} *$," Journal of Biological Chemistry, vol. 274, no. 14, pp. 98289835, 1999.

[68] A. Yamaguchi, M. Tamatani, H. Matsuzaki et al., "Akt activation protects hippocampal neurons from apoptosis by inhibiting transcriptional activity of p53," Journal of Biological Chemistry, vol. 276, no. 7, pp. 5256-5264, 2001.

[69] M. McMillin, G. Frampton, S. Grant, F. Sohrabji, F. Meng, and S. DeMorrow, "TGF $\beta 1$ suppresses neuronal IGF1 via a let-7Fdependent mechanism during azoxymethane-induced hepatic encephalopathy in mice," Journal of Clinical and Experimental Hepatology, vol. 7, pp. S11-S12, 2017.

[70] R. H. Garman, "Histology of the central nervous system," Toxicologic Pathology, vol. 39, no. 1, pp. 22-35, 2011. 